\title{
High Transaminases Following Cardiac Surgery: A Narrative Review
}

\author{
Amr S. Omar ${ }^{1,2,3}$ Adel Taha ${ }^{4} \quad$ Abdulaziz AlKhulaifi \\ ${ }^{1}$ Department of Cardiothoracic Surgery/Cardiac Anesthesia and \\ ICU, Heart Hospital, Hamad Medical Corporation, Doha, Qatar \\ ${ }^{2}$ Department of Critical Care Medicine, Beni Suef University, \\ Beni Suef, Egypt \\ ${ }^{3}$ Department of Clinical Medicine, Weill Cornell Medical College, \\ Qatar \\ ${ }^{4}$ Department of Critical Care Medicine, King Khaled Specialized \\ Hospital, Dammam, KSA
}

J Card Crit Care:2020;4:111-115

\begin{abstract}
Address for correspondence Amr S. Omar, MD, PhD, MBA, Department of Cardiothoracic Surgery/Cardiac Anesthesia and ICU Section, Heart Hospital, Hamad Medical Corporation, Doha, PO, 3050, Qatar (e-mail: a_s_omar@yahoo.com).
\end{abstract}

\begin{abstract}
Elevation of liver enzymes after cardiac surgery is encountered infrequently. Acute heart failure during and after surgery may be the culprit responsible for liver dysfunction. However, it may create clinical confusion whether acute liver dysfunction could induce some sort of cardiac dysfunction through mechanisms similar to those encountered in chronic liver disease. We searched through the Medline, Cochrane, and Embase databases up to January 2018. We included review articles, metaanalyses, and original trials on the elevation of liver enzymes after cardiac surgery, and combined the following MESH terms: "intensive care, "cardiac surgery," "high liver enzymes," "ischemia," "left ventricular dysfunction," and "critical illness." Case reports were excluded. Language restrictions were not applied. References were examined for

Keywords

- transaminases

- cardiac surgery

- cardiac function

- high liver enzymes other potentially useful articles. We did not find any articles that supported the cardiac decompensation phenomenon after acute liver injury. In contrast, low-hepatic flow, hypoxemia, or pump-induced inflammation could induce hepatic dysfunction in acute settings after cardiac surgery. In conclusion, a rise in the transaminases following cardiac surgery would favor an ischemic etiology for the condition.
\end{abstract}

\section{Introduction}

Elevation of liver function tests (LFT) is an infrequent complication after cardiac surgery with an incidence of less than $1 \%{ }^{1}$ This complication plays a pivotal role in overall intensive care unit outcome, as it is associated with a series of hemodynamic, metabolic, inflammatory derangement that could affect the overall morbidity and mortality. ${ }^{2}$ In acute settings, the cardiac causes of elevated LFTs included hypotension, low-cardiac output, right ventricular failure, intravascular volume depletion, and vasodilatation. The reason for the elevation in LFTs may not be clear. ${ }^{3}$ Cardiac hepatopathy exists in two main forms which are acute cardiogenic liver injury

published online

September 10, 2020
DOI https://doi.org/

$10.1055 / \mathrm{s}-0040-1715931$

ISSN 2457-0206. and congestive hepatopathy. The former associates acute heart failure, while the latter comes about in the settings of right-sided heart failure as a consequence of passive venous congestion; the two situations may coexist and result in increase in the liver enzyme. ${ }^{4,5}$

Chronic liver disease is a state that may participate and accelerate impairment of cardiac functions and cardiac remodeling. Cirrhotic patients have 45 to $56 \%$ prevalence of diastolic dysfunction, while the latter is reflected in some degree by left atrial enlargement. Cardiomyopathy in patients with chronic liver disease could be boosted by many mechanisms, including $\beta$-adrenergic receptor pathway signaling inhibition,

(c) 2020. Official Publication of The Simulation Society (TSS), accredited by International Society of Cardiovascular Ultrasound (ISCU).

This is an open access article published by Thieme under the terms of the Creative Commons Attribution-NonDerivative-NonCommercial-License, permitting copying and reproduction so long as the original work is given appropriate credit. Contents may not be used for commercial purposes, or adapted, remixed, transformed or built upon. (https://creativecommons.org/licenses/by-nc-nd/4.0/)

Thieme Medical and Scientific Publishers Pvt. Ltd., A-12, 2nd Floor, Sector 2, Noida-201301 UP, India 
cytoplasmic as well as nuclear cardiomyocytes vacuolation and, finally, cardiomyocyte cellular membrane changes. ${ }^{6}$

The cardiac hepatic interaction is crucial for cardiologists, hepatologists, and intensivists. ${ }^{7}$ Chronic liver diseases may induce a clinical picture of cardiomyopathy; in chronic hepatitis $C$, viral infection could stimulate proliferation of myocytes with subsequent hypertrophy and hypertrophic cardiomyopathy. ${ }^{8}$ In patients with liver cirrhosis, the sympathetic nervous system activity is augmented and cardiac output is increased due to hyperdynamic circulation and reduced systemic vascular resistance. The latter changes could prompt left ventricular hypertrophy and myocardial remodeling that lead to disordered systolic and diastolic functions, and cardiomyopathy. ${ }^{9}$ Chronic liver diseases may induce systolic and diastolic dysfunction in addition to prolongation of QT interval and electrophysiological alterations, given the changes in cirrhotic cardiomyopathy may recover completely following liver transplantations.

Causes of elevation of liver enzymes

\section{Postsurgical}

Elevation in LFTs more than five-fold, which associates with increase in conjugated bilirubin, may reflect hepatocellular injury that occurs in hepatic hypoxia and drug-induced hepatitis. ${ }^{2}$

2. Myocardial dysfunction

The low-cardiac output that follows heart failure, acute myocardial infarction, and pericarditis may result in liver dysfunction, owing to reduced blood supply to the liver and passive hepatic venous congestion. The latter is characterized by moderate elevation of LFTs < three-fold in 30 to $60 \%$ of cases, bilirubin elevation up to ten-fold in 25 to $80 \%$ of patients, and prothrombin time (2-6 seconds) in 80 to $90 \%{ }^{10}$

Cardiac arrest or arrhythmia-induced hypotension can result in hypoxic hepatitis, with striking elevation in LFTs. ${ }^{5}$ Cardiopulmonary bypass (CPB) surgery may also induce liver dysfunction. ${ }^{11}$ Off pump coronary bypass decreases the possibility of hepatocellular damage induced by CBP. ${ }^{12}$

3. Sepsis

Reperfusion injury and hepatic ischemia may follow sepsis. ${ }^{9}$ Septic shock is associated with major metabolic demand increment, where tissue oxygen consumption and oxygen extraction are affected. ${ }^{13}$ Oxygen consumption increment could also be associated with cytokine production and the deviation of oxygen to the generation of reactive oxygen species. ${ }^{14}$ In addition, hepatic flow may be reduced due to the effect of mechanical ventilation from increased intrathoracic pressure. ${ }^{15}$

Hepatic microcirculation is affected and the LFTs deteriorate gradually after sepsis. ${ }^{16}$ Complex interaction does occur between pro- and anti-inflammatory mediators, aiming to maintain normal arterial pressure and protect liver tissue; the severity of hepatic injury is determined by Kupffer cells in shock situations. ${ }^{17}$

4. Hypoxic hepatitis

The dual liver blood supply form the hepatic artery and portal vein attempt to maintain constant hepatic flow even in shock status. Dilatation of hepatic artery occurs in response to reduced portal venous flow (in the range of $20-30 \%) .{ }^{18}$ The oxygen content in the hepatic artery is higher than the portal vein, which made oxygen compensation substantially better. ${ }^{19}$ Cellular ischemic is produced if hepatic microcirculation ceases under a critical level, resulting in hepatic injury and dysfunction. At the same time, nitric oxide (NO) seeks to maintain endothelial integrity and hepatic microcirculation, which is the result of the insult. ${ }^{20}$ Pericentral necrosis and ATP depletion is induced by extended low-flow with hypoxia, where regeneration of oxygen and ATP supplies after shorter-term, low-flow ischemia reproduces programmed cell death. ${ }^{21}$ In heart failure, liver hypoxia is referred to as reduced hepatic blood flow in left-sided heart failure, and as venous congestion in right-sided heart failure. The rapid increase in LFTs that accompany hypoxia usually leads to recovery within a few days. ${ }^{22}$

5. Acute respiratory distress syndrome (ARDS)

Liver dysfunction may accompany ARDS; however, on the other hand, hepatic injury association significantly impairs outcome in critically ill adults. ${ }^{23}$

6. Intervention induced liver dysfunction

Medical and surgical procedures in critically ill patients may be associated with abnormalities in LFTs. Ultrafiltration and hemodialysis could be associated with hepatic hypoxia from reduced splanchnic, femoral, and systemic blood flows. ${ }^{24}$ The critically ill have commonly tied physiological reserve with altered variation in drug metabolism and higher chances of adverse events. ICU medication induced by hepatitis is common and may be due to inotropes. Hepatic injury form systemic arterial blood flow changes may include but not be limited to granulomatous hepatitis (hydralazine and quinidine), acute hepatitis (amiodarone, diltiazem, b-blockers, lisinopril, and hydralazine), fibrosis (amiodarone), cholestasis (nifedipin and disopyramide), or mixed changes (procainamide). ${ }^{2}$

\section{Results}

We did not find any literature to support the cardiac decompensation nature, following acute liver insults. In contrary, low-hepatic flow, hypoxemia, or pump-induced inflammation could induce hepatic dysfunction in acute settings after cardiac surgery.

\section{Discussion}

An argument exists as to whether the liver dysfunction may aggravate the cardiac dysfunction as a part of systemic affection in acute settings. In sepsis-induced cardiomyopathy, model dilatation of both ventricles could occur with associated reduction in ejection fraction, which is reflected in fluid resuscitation and poor response of catecholamines. ${ }^{25}$ Sepsisinduced cardiomyopathies have led physicians to hypothesize about the possible systemic nature of myocardial dysfunction in acute hepatic events. Raman et al (-Table 1) described liver injury after cardiac surgery and called it severe ischemic early liver injury (SIELI). ${ }^{26}$ The authors noted 
Troponin, Cardiac Surgery, High Liver Enzymes Omar et al. 113

\begin{tabular}{|c|c|c|c|c|c|c|c|c|c|}
\hline & 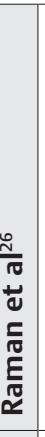 & 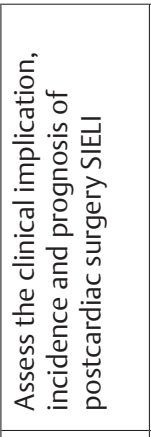 & 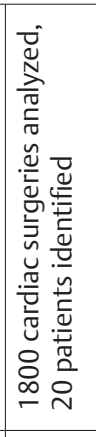 & 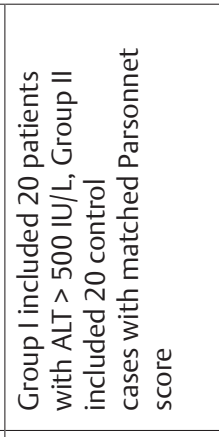 & 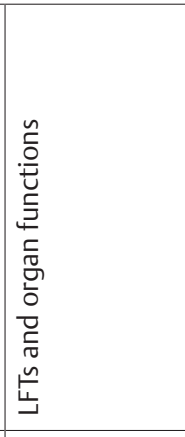 & 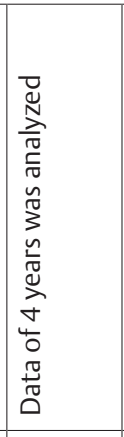 & 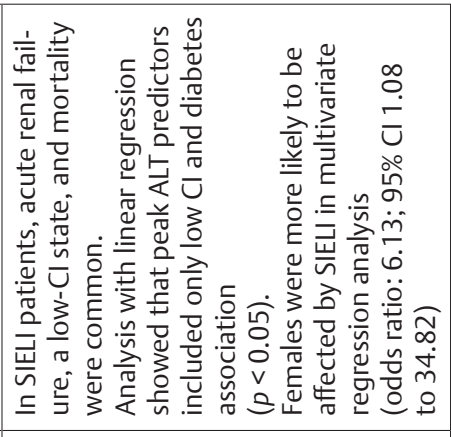 & 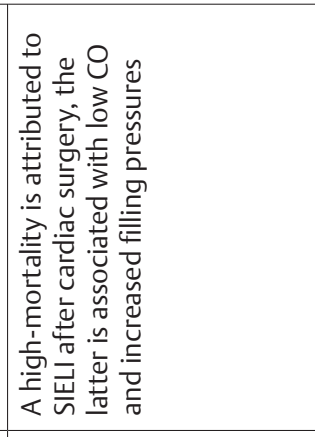 & 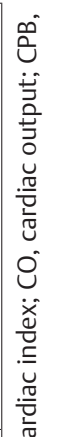 \\
\hline & 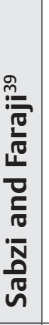 & 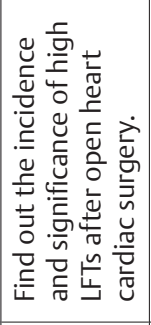 & 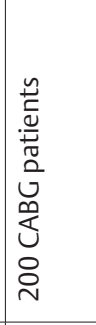 & 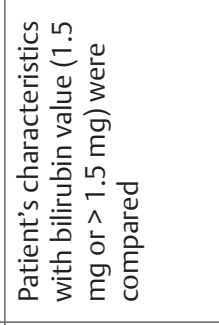 & 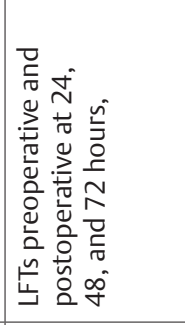 & 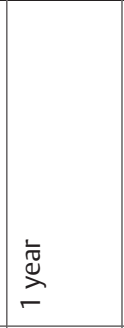 & 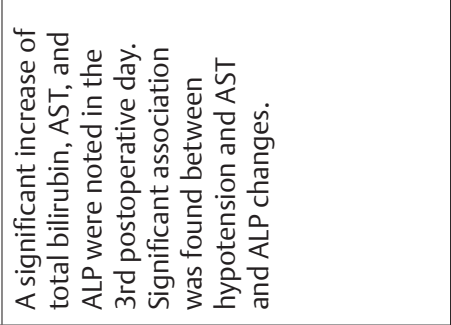 & 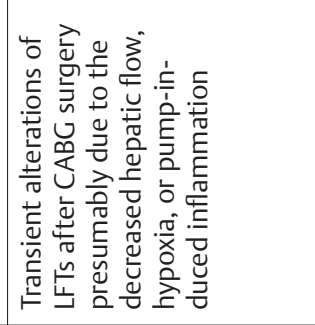 & 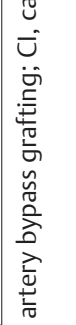 \\
\hline & 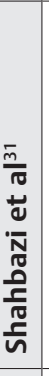 & 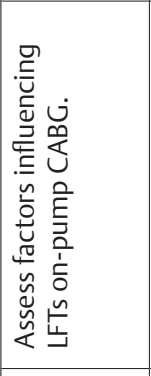 & 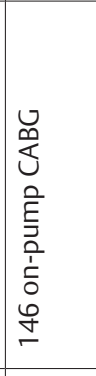 & 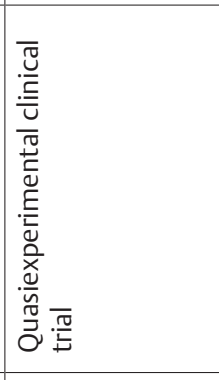 & 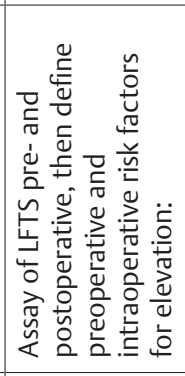 & 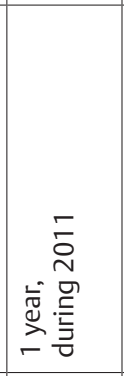 & 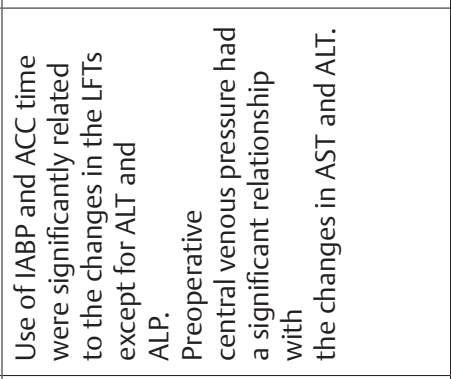 & 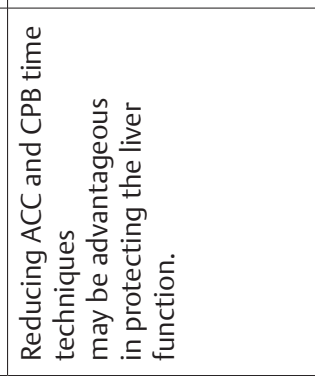 & \\
\hline & 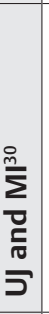 & 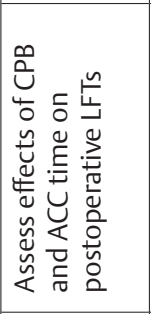 & 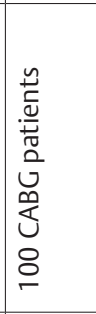 & 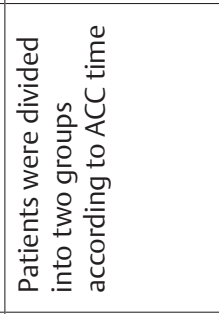 & 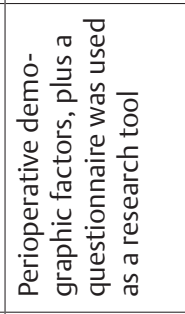 & 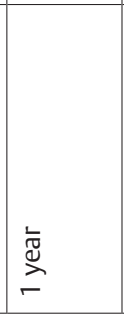 & 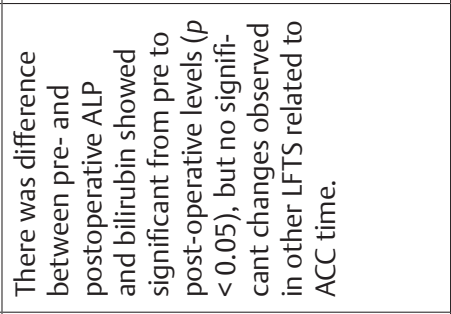 & 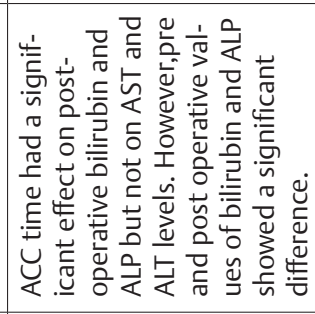 & \\
\hline & 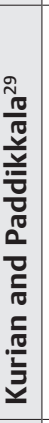 & 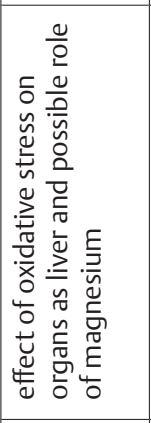 & 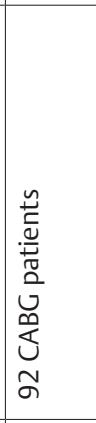 & 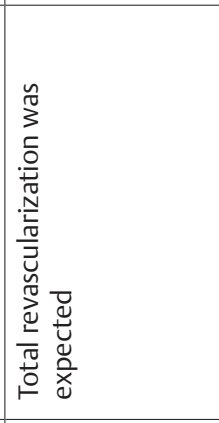 & 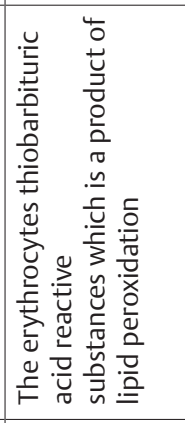 & 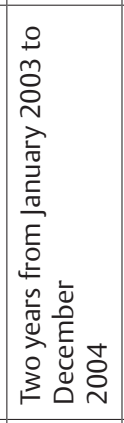 & 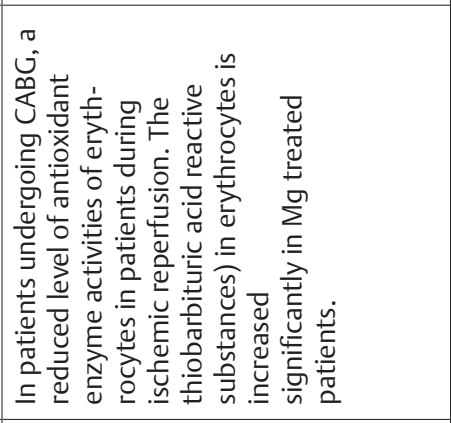 & 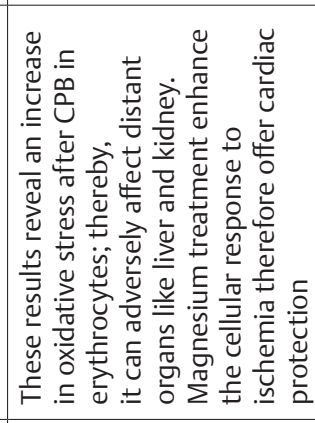 & \\
\hline & 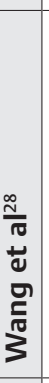 & 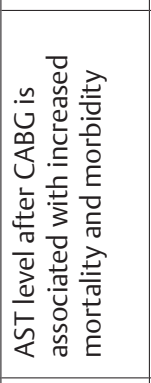 & 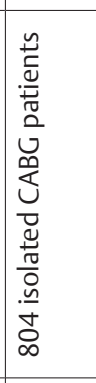 & 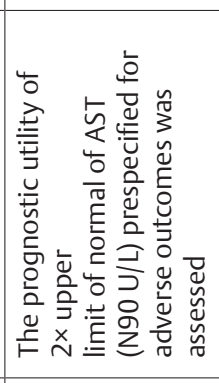 & 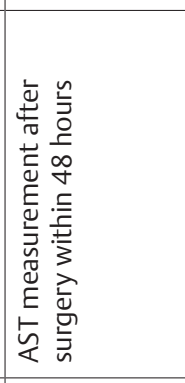 & 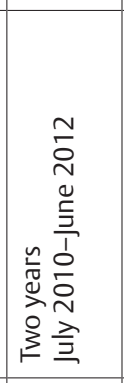 & 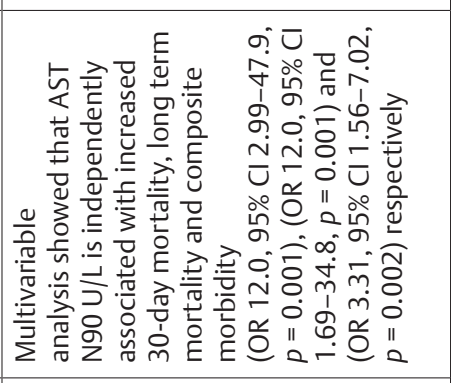 & 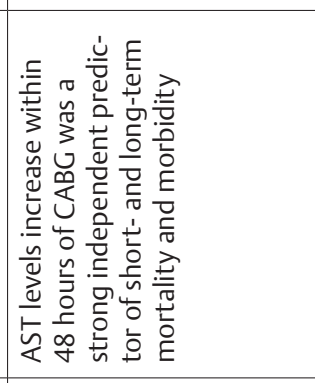 & \\
\hline & 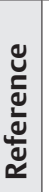 & 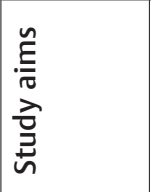 & 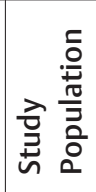 & 罢 & 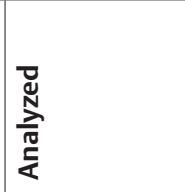 & 은 & $\frac{\mathscr{n}}{\overrightarrow{\breve{y}}}$ & 竞 & \\
\hline
\end{tabular}


the high-mortality rate in this group and associated morbidity, including acute kidney injury and low-cardiac index. The hemodynamic profile included higher rates of peak postoperative pulmonary artery occlusion (PAOP) and central venous pressure (CVP) with higher demands of norepinephrine and milrinone. Liver ischemia following cardiac surgery could be driven by an integration of congestion and decreased perfusion. ${ }^{26}$ The liver's unique dual blood supply offers a proportional guard against ischemic injury; however, ischemia and necrosis are more common in hepatocytes in zone 3 due to low-oxygen tension in the microcirculation (sinusoidal blood of the hepatic acinus). ${ }^{27}$

Wang et al emphasized the predictive power of aspartate aminotransferase (AST) in predicting morbidity and mortality after cardiac surgery. ${ }^{28}$ There is an increase in oxidative stress after $\mathrm{CPB}$ in erythrocytes; therefore, it can adversely affect distant organs like liver. ${ }^{29}$ Aortic cross-clamp (ACC) may have a significant effect on postoperative bilirubin and alkaline phosphatase (ALP). ${ }^{30}$ Shahbazi et $\mathrm{al}^{31}$ stated that reducing the CPP and ACC times may abort the raise in liver functions ( - Table $\mathbf{1}$ ).

There is no published research to support the theory that acute liver dysfunction induces acute changes in cardiac muscle function. On the contrary, acute heart failure emerges in settings of marked systemic hypotension which is associated with acute cardiopulmonary collapse. ${ }^{32}$ Despite the obscurity of profound hypotension, severe hypoxemia could be the culprit behind ischemic hepatitis. ${ }^{33}$ Centrilobular necrosis of zone 3 hepatocytes characterizes ischemic liver injury. Histological evidence of inflammation indicative of viral hepatitis is absent. ${ }^{34}$ Reduced hepatic blood flow is associated with increased oxygen consumption, but when tissue hypoxia is persistent and end-organ perfusion is inappropriate or when acute shock evolves, reduced hepatic flow exhausts the protecting mechanism against hypoxic liver damage. Liver injury supervenes, which is associated with a sharp elevation of serum AST, alanine aminotransferase (ALT), lactic dehydrogenase (LDH), and prothrombin time prolongation. These changes peak within 1 to 3 days and revert subsequently. The unique dual liver blood supply may be restored back to normal within 5 to 10 days following cardiogenic ischemic hepatitis. $^{35}$ Ischemic liver injuries can be differentiated from other forms of acute hepatitis by a marked increase in LDH levels, associated with ALT/LDH ratio of less than 1.5. ${ }^{36}$

Assuming that impaired cardiac function, which is secondary to liver affection after cardiac surgery, does not hold true position unlike in sepsis model where sepsis-induced cardiomyopathy produces impairment of left and right ventricular functions without being selective to one chamber. ${ }^{37}$ However, any cause of right ventricular dysfunction can be associated with severe hepatic congestion; patients with hepatic congestion are usually asymptomatic and congestion may be indicated only by abnormal LFTs during routine laboratory analysis. The primary pathophysiology involved in hepatic dysfunction is either passive congestion from increased filling pressures or low-cardiac output and the consequences of impaired perfusion. ${ }^{37}$ Interestingly, in the Raman study, the peak postoperative ALT levels correlated significantly with pulmonary artery occlusion and central venous pressures, and negatively with cardiac index. ${ }^{11}$ The factors that raise a propensity for liver injury after cardiac surgery include the amount of blood transfused, number of valves replaced, existing chronic hepatic congestion, hypothermia, lowblood supply, hemolysis, sepsis, and renal failure. ${ }^{2}$ The LFTs could be affected also by the duration of the ACC and use of an intra-aortic balloon pump (IABP). ${ }^{31}$ The possibility of statin-induced acute elevation of LFTs after cardiac surgery is remote..$^{38}$ Finally, the transient elevation of LFTs following a coronary artery bypass grafting could be attributed to the decreased hepatic flow, hypoxia, or pump-induced inflammation. ${ }^{39}$

\section{Conclusion}

Introducing transaminases for postoperative cardiac surgeries risk assessments represent an important paradigm in perioperative medicine. Assessments of transaminases provide risk prediction and help to allocate resources in the right direction of patient care. Hepatic dysfunction-induced cardiac dysfunction is known in chronic liver conditions, but its value in acute settings needs better understanding. We concluded that $s$ rise in the transaminases after cardiac surgery would favor the ischemic nature of the condition. We anticipate that ongoing research on risk stratifying and treating patients with high transaminases will further improve outcomes for patients undergoing cardiac surgery.

\section{Authors' Contributions}

ASO initiated the idea, wrote the manuscript, designed the study, and submitted the manuscript; AKT, as chair of the intensive care department, offered critical revision provided general support. All authors read and approved the final manuscript.

\section{Financial Competing Interests}

I did not receive reimbursements, fees, funding, or salary from an organization in the past 5 years which may in any way gain or lose financially from the publication of this manuscript. However, Hamad medical corporation is going to fund the article processing charges.

I do not hold any stocks or shares in an organization which may in any way gain or lose financially from the publication of this manuscript either now or in the future.

I do not hold and I am not currently applying for any patents relating to the content of the manuscript. I did not receive reimbursements, fees, funding, or salary from an organization that holds or has applied for patents relating to the content of the manuscript.

I do not have any other financial competing interests. There are no other nonfinancial competing interests (political, personal, religious, ideological, academic, intellectual, commercial, or any other) to declare in relation to this manuscript.

\section{Conflicts of Interest}

None declared. 


\section{Acknowledgments}

This project would not have been possible without the unlimited help and support of many individuals and our organization. The authors would like to thank all members of the cardiothoracic surgery department, Heart Hospital, of Hamad Medical Corporation, Qatar, for providing all the required data related to this work. The authors would also like to extend their gratitude to the medical research department of Hamad Medical Corporation for their continuous support throughout the course of this project.

\section{References}

1 Michalopoulos A, Alivizatos P, Geroulanos S. Hepatic dysfunction following cardiac surgery: determinants and consequences. Hepatogastroenterology 1997;44(15):779-783

2 Soultati A, Dourakis A. Liver dysfunction in the intensive care unit. Ann Gastroenterol 2007;18(1):35-45

3 Bernal W, Wendon J. Acute liver failure. $\mathrm{N}$ Engl J Med 2013;369(26):2525-2534

4 Çağlı K, Başar FN, Tok D, Turak O, Başar Ö. How to interpret liver function tests in heart failure patients? Turk J Gastroenterol 2015;26(3):197-203

5 Møller S, Bernardi M. Interactions of the heart and the liver. Eur Heart J 2013;34(36):2804-2811

6 Poelzl G, Auer J. Cardiohepatic syndrome. Curr Heart Fail Rep 2015;12(1):68-78

7 Fouad YM, Yehia R. Hepato-cardiac disorders. World J Hepatol 2014;6(1):41-54

8 Matsumori A. Hepatitis C virus infection and cardiomyopathies. Circ Res 2005;96(2):144-147

9 Møller S, Henriksen JH. Cirrhotic cardiomyopathy: a pathophysiological review of circulatory dysfunction in liver disease. Heart 2002;87(1):9-15

10 Henrion J, Descamps O, Luwaert R, Schapira M, Parfonry A, Heller F. Hypoxic hepatitis in patients with cardiac failure: incidence in a coronary care unit and measurement of hepatic blood flow. J Hepatol 1994;21(5):696-703

11 Collins JD, Bassendine MF, Ferner R, et al. Incidence and prognostic importance of jaundice after cardiopulmonary bypass surgery. Lancet $1983 ; 1(8334): 1119-1123$

12 Okano N, Hiraoka H, Owada R, et al. Hepatosplanchnic oxygenation is better preserved during mild hypothermic than during normothermic cardiopulmonary bypass. Canadian journal of anaesthesia. 2001 Nov 1;48(10):1011-1014

13 Clemmesen O, Ott P, Larsen FS. Splanchnic metabolism in acute liver failure and sepsis. Curr Opin Crit Care 2004;10(2):152-155

14 Jakob SM. Clinical review: splanchnic ischaemia. Crit Care 2002;6(4):306-312

15 James PE, Madhani M, Roebuck W, Jackson SK, Swartz HM. Endotoxin-induced liver hypoxia: defective oxygen delivery versus oxygen consumption. Nitric Oxide 2002;6(1):18-28

16 Hwang TL, Han ML. The changes of hepatic sinusoidal microcirculation and effects of nitric oxide synthase inhibitor during sepsis. Hepatogastroenterology 2003;50(49):213-216

17 Fang C, Yoon S, Tindberg N, Järveläinen HA, Lindros KO, Ingelman-Sundberg $M$. Hepatic expression of multiple acute phase proteins and down-regulation of nuclear receptors after acute endotoxin exposure. Biochem Pharmacol 2004;67(7):1389-1397

18 Lautt WW. Mechanism and role of intrinsic regulation of hepatic arterial blood flow: hepatic arterial buffer response. Am J Physiol 1985;249(5 Pt 1) :G549-G556
19 Rasmussen A, Skak C, Kristensen M, Ott P, Kirkegaard P, Secher NH. Preserved arterial flow secures hepatic oxygenation during haemorrhage in the pig. J Physiol 1999;516(Pt 2): 539-548

20 Chen T, Zamora R, Zuckerbraun B, Billiar TR. Role of nitric oxide in liver injury. Curr Mol Med 2003;3(6):519-526

21 Paxian M, Bauer I, Rensing H, et al. Recovery of hepatocellular ATP and "pericentral apoptosis" after hemorrhage and resuscitation. FASEB J 2003;17(9):993-1002

22 Weisiger RA, Laboratory tests in liver disease. In: Bennet JC, Plum F, eds. Cecil Textbook of Medicine. 20th ed. London: WB Saunders Company; 1996 ; 759-762

23 McIntyre RC Jr, Pulido EJ, Bensard DD, Shames BD, Abraham E. Thirty years of clinical trials in acute respiratory distress syndrome. Crit Care Med 2000;28(9):3314-3331

24 Jakob SM, Ruokonen E, Vuolteenaho O, Lampainen E, Takala J. Splanchnic perfusion during hemodialysis: evidence for marginal tissue perfusion. Crit Care Med 2001;29(7):1393-1398

25 Romero-Bermejo FJ, Ruiz-Bailen M, Gil-Cebrian J, HuertosRanchal MJ. Sepsis-induced cardiomyopathy. Curr Cardiol Rev 2011;7(3):163-183

26 Raman JS, Kochi K, Morimatsu H, Buxton B, Bellomo R. Severe ischemic early liver injury after cardiac surgery. Ann Thorac Surg 2002;74(5):1601-1606

27 Lautt WW, Greenway CV. Conceptual review of the hepatic vascular bed. Hepatology 1987;7(5):952-963

28 Wang TK, Stewart RA, Ramanathan T, Gamble G, White HD. Post-operative aspartate aminotransferase levels independently predict mortality after isolated coronary artery bypass grafting. IJC Metab Endocr 2015;6:31-35

29 Kurian GA, Paddikkala J. Biochemical changes in liver and kidney functions of patients subjected to revascularization in CABG procedure: role of magnesium supplementation. Appl Cardiopulm Pathophysiol 2010;14:29-39

30 UJ II. MI H. Effect of cardiopulmonary bypass on liver function in patients undergoing coronary artery bypass grafting. Biomedica 2015;31(1):27

31 Shahbazi S, Panah A, Sahmeddini MA. Evaluation of factors influencing liver function test in on-pump coronary artery bypass graft surgery. Iran J Med Sci 2013;38(4):308-313

32 Lefkowitch JH, Mendez L. Morphologic features of hepatic injury in cardiac disease and shock. J Hepatol 1986;2(3):313-327

33 Henrion J, Minette P, Colin L, Schapira M, Delannoy A, Heller FR. Hypoxic hepatitis caused by acute exacerbation of chronic respiratory failure: a case-controlled, hemodynamic study of 17 consecutive cases. Hepatology 1999;29(2):427-433

34 Ellenberg M, Osserman KE. The role of shock in the production of central liver cell necrosis. Am J Med 1951;11(2):170-178

35 Alvarez AM, Mukherjee D. Liver abnormalities in cardiac diseases and heart failure. Int J Angiol 2011;20(3):135-142

36 Cassidy WM, Reynolds TB. Serum lactic dehydrogenase in the differential diagnosis of acute hepatocellular injury. J Clin Gastroenterol 1994;19(2):118-121

37 Parker MM, McCarthy KE, Ognibene FP, Parrillo JE. Right ventricular dysfunction and dilatation, similar to left ventricular changes, characterize the cardiac depression of septic shock in humans. Chest 1990;97(1):126-131

38 Aboulnaga S, Mahfouz A, Ewila HA, et al. Postoperative cardiac surgery outcomes in a statin-native population. Anesthesia, Essays and Researches 2018;12(1):223

39 Sabzi F, Faraji R. Liver function tests following open cardiac surgery. J Cardiovasc Thorac Res 2015;7(2):49-54 